Sección uno: Ensayo

Radiografía de la innovación educativa en el Siglo XXI

\title{
Innovación en los sistemas de evaluación del aprendizaje basado en proyectos ${ }^{1}$
}

Innovation in project based learning evaluation systems

\author{
María Encarnación Carrillo-García \\ Universidad de Murcia \\ mariaencarnacion.carrillo@um.es \\ Antonia Cascales-Martínez \\ Universidad de Murcia \\ antonia.cascales@um.es
}

\section{Resumen}

Los objetivos de esta investigación son dos: conocer los recursos y técnicas de evaluación que se utilizan cuando el profesorado trabaja con el Aprendizaje Basados en Proyectos (ABP); e identificar las diferencias sociodemográficas, estadísticamente significativas, sobre el sistema de evaluación en el ABP. El método exploratorio utilizado fue del tipo cuantitativo no experimental, mediante un cuestionario diseñado ad hoc con la finalidad de conocer las opiniones acerca de la evaluación en el ABP, y el número de profesores y profesoras que participaron fue 43. Los resultados nos llevaron a concluir que el profesorado considera importante la evaluación del proceso de enseñanza y aprendizaje con esta metodología, así como el uso de diferentes instrumentos de evaluación, que ayuden a evaluar a corto y largo plazo, así como a valorar el trabajo del equipo; y entre los instrumentos de evaluación: las rúbricas están muy bien valoradas, especialmente entre mujeres y profesorado más joven.

Palabras clave: enseñanza; evaluación; innovación; método de aprendizaje.

\footnotetext{
${ }^{1}$ Recibido: 05/12/2019 Evaluado: 04/01/2020 Aceptado: 02/04/2020
} 


\begin{abstract}
The objectives of this research are: to know the resources and evaluation techniques that are used when teachers work with ProjectBased Learning (PBL), and identify the statistically significant sociodemographic differences on the evaluation system in the PBL. The exploratory method used was a non-experimental quantitative type, through a questionnaire designed ad hoc in order to know the opinions about the evaluation in the PBL, and the number of teachers who participated was 43. The results led us to conclude that teachers consider the evaluation of the teaching and learning process with this methodology important, as well as the use of different evaluation instruments, which help to evaluate in the short and long term, as well as to evaluate the work of the team; and among the evaluation instruments, the rubrics are very well valued, especially among women and younger teachers.
\end{abstract}

Keyword: teaching; evaluation; innovation; learning method.

\title{
Fundamentación teórica
}

\section{Evaluando procesos y resultados}

El desafío que presenta la evaluación del Aprendizaje Basado en Proyectos (ABP) en comparación con otros métodos pedagógicos lleva a autores como Sa, Ezenwaka, Singh, Vuma y Majumder (2019) a declarar que el mayor reto en los procesos de enseñanza y aprendizaje en ABP está en la evaluación de su proceso, donde el alumnado no solo va a conseguir almacenar contenidos teóricos, si no que va a adquirir destrezas, habilidades y competencias que lo lleven a aplicar dichos contenidos en la práctica, a través de los proyectos que se le propongan. Según Utech (2003), las evaluaciones tradicionales no pueden medir habilidades tales como trabajar en equipo, realizar investigaciones o describir un problema y presentar una solución razonable; dado que se entiende por evaluación tradicional aquella que está supeditada a la norma mediante un único procedimiento y estrategia siendo el propietario exclusivo el docente (Tobón, 2006), en la que el docente evalúa el resultado de los aprendizajes mediante una única prueba exploratoria, como pudiera ser un modelo tipo test o examen, donde se califica en función de las respuestas correctas; sin embargo, en la evaluación en el ABP, el profesorado debe tener en cuenta aquellos elementos que forman parte del proceso de resolución del problema presentado, y no solo la solución final. Coincidimos con Sa et al. (2019) en que la evaluación es uno de los elementos más importantes que el profesorado debe tener en cuenta al aplicar metodologías innovadoras y activas, donde el papel del alumnado como responsable de su propio proceso de aprendizaje debe adquirir también un papel activo en cuanto a la evaluación se refiere. Autores como Shafirova y Cassany (2017) defienden propuestas didácticas que fomentan el aprendizaje activo como forma de aprender una lengua usándola, premisa que cumple el ABP, ya que es diferente a otras estrategias pedagógicas de corte tradicional donde el alumnado es un sujeto pasivo expuesto a los contenidos por parte del profesorado(Cascales-Martínez, CarrilloGarcía y Redondo-Rocamora 2017), pero no hay que olvidar que la evaluación también debe atenerse a otras premisas, y debe incluir como elementos a evaluar aquellos aspectos que hacen diferente al ABP, donde el rol del alumnado y del profesorado se transforman, 
convirtiéndose uno en agente responsable de su propio aprendizaje y el otro en guía y consejero del proceso de enseñanza y aprendizaje respectivamente (Cascales-Martínez y Carrillo-García, 2018). Esta forma de plantear la evaluación en el ABP lejos de presentarse como una dificultad debe ser entendida como un reto, que aunque no esté exento de dificultad debe ser un estímulo para el profesorado que quiera mejorar sus competencias a nivel de evaluación en el ABP. Debido a que hay algunos elementos a evaluar y que son centrales para el ABP, relacionados con el desarrollo del proceso de investigación, práctica, producción y creación de contenidos por parte del alumnado, y que la evaluación tradicional generalmente ha descuidado evaluar, es necesario realizar una revisión que enfoque la evaluación en cada uno de los aspectos trabajados con este planteamiento didáctico.

Como vemos, la razón principal de la dificultad de la evaluación en el ABP surge de las características de este enfoque metodológico. Cuando el profesorado tiene que evaluar preguntas que solo tienen una respuesta correcta posible, el proceso de evaluación es sencillo, ya que solo se trata de evaluar una respuesta correcta o incorrecta. Pero cuando hay varias respuestas correctas posibles a la misma pregunta, y hay cierto gradiente de precisión en las respuestas posibles, el proceso de evaluación se vuelve mucho más complicado; y coincidimos con Giabbanelli y Tawfik, (2017) que es un desafío para el profesorado evaluar múltiples respuestas pertenecientes a un mismo proyecto; es por ello necesario que el profesorado centre su foco de atención en los procesos de evaluación, incluyéndolos dentro del proceso de enseñanza y aprendizaje, de manera que su integración también lleve a un mayor nivel de adquisición del conocimiento por parte del alumnado.

Por otro lado, es importante matizar que mientras que las formas tradicionales de evaluación miden el conocimiento de los estudiantes, la evaluación ABP debe evaluar el proceso de aplicación de ese conocimiento en situaciones de la vida real. Es por tanto que nos encontramos con otro reto cuando se evalúan proyectos relacionados con cuestiones del mundo real, porque tal y como señala Utech (2003) este elemento hace que el uso de la evaluación tradicional sea inadecuado, ya que, si bien las evaluaciones tradicionales miden el conocimiento del alumnado, no logra establecer una conexión con la forma en que dicho alumnado puede usar este conocimiento en un contexto de uso auténtico. En otras palabras, esta es una cuestión que el profesorado debe afrontar con el objetivo de diseñar e implementar una adecuada evaluación en este complejo proceso educativo, que no por ello debe calificarse de difícil, como es el ABP, sino un proceso que conlleva una evaluación a diferentes niveles de contenidos procedimentales, conceptuales y actitudinales.

\section{Técnicas e instrumentos de evaluación en el ABP}

Como hemos visto anteriormente, para superar estas dificultades, la evaluación en el ABP debe medir la comprensión, no la memorización; la capacidad del alumno para resolver un problema, no solo una respuesta correcta o incorrecta; y la capacidad del estudiante para trabajar como miembro de un equipo (Gentry, 2000), elementos todos ellos que no se tienen en cuenta en los métodos tradicionales de evaluación donde se evalúan contenidos conceptuales pero, como son elementos centrales de ABP deben evaluarse. Para evaluar todos estos elementos, el profesorado puede incluir una variedad de instrumentos de evaluación como son portafolios, diarios, trabajos periódicos y otros recursos para evaluar el cumplimiento de los objetivos a corto y largo plazo (Gentry, 2000). En otras palabras, se 
deben incorporar los procedimientos de evaluación que se consideren útiles para evaluar no solo la solución final del problema, sino todo el proceso que los estudiantes han seguido para llegar a sus conclusiones.

Debido a todo eso con el fin de optimizar el proceso de evaluación, en la evaluación de ABP se debe incluir no solo una evaluación por parte del profesorado, sino también una evaluación por pares, una autoevaluación (Gentry, 2000) e incluso una evaluación pública con una audiencia de expertos (Thomas, 2000). Es muy importante destacar que, incluir a los estudiantes en el proceso de evaluación contribuye a su autonomía, que es un elemento importante que debe desarrollarse con la enseñanza de ABP; y ayuda al profesorado a evaluar ciertos elementos que quizás sean más fáciles de evaluar por los estudiantes que por él. Los elementos de autoevaluación también pueden ser útiles para que los estudiantes se den cuenta del progreso que han realizado durante el proyecto, particularmente si se incluye una autoevaluación inicial antes de que se inicie el proyecto y otra autoevaluación cuando el proyecto esté terminado.

Como hemos visto, hay muchos elementos para evaluar cuando se lleva a cabo un proyecto, y hay muchos instrumentos y métodos que pueden usarse para evaluar esos proyectos. Todos estos elementos hacen que la evaluación de ABP sea muy desafiante, particularmente si se va a lograr un alto grado de confiabilidad. Según Brodie y Gibbins (2019), es muy difícil garantizar estándares de evaluación consistentes, particularmente cuando los elementos de evaluación son abiertos, en el sentido de que permiten dar por válidas más de una respuesta o ejecución de un mismo proyecto. En muchas ocasiones puede haber un alto grado de subjetividad en la evaluación de ABP, porque hay muchos elementos que el profesorado debe considerar, y sobre todo cuando no hay una respuesta única a los interrogantes que el alumnado tiene que resolver. La influencia del evaluador o evaluadora es, por lo tanto, notable, porque los factores humanos como el sesgo personal, los errores como el efecto de indulgencia, el efecto estricto, el error de tendencia central, el error lógico y el efecto halo pueden afectar la calificación de los tutores de los estudiantes en ABP (Sa et al., 2019); si bien, esto es en realidad un problema que está presente en todos los enfoques pedagógicos cuando el profesorado debe evaluar elementos que no son claramente objetivos.

Como la fiabilidad y la coherencia son elementos cruciales para lograr un cierto grado de equidad en la evaluación del alumnado, se deben encontrar algunos instrumentos para ayudar al profesorado a alcanzar cierto nivel de objetividad en la evaluación del ABP. Para superar estos problemas, lo deseable es encontrar un método de calificación apropiado justo y equitativo. Las rúbricas de puntuación o marcado a menudo se usan para esta tarea (Brodie y Gibbins, 2019). Particularmente, las rúbricas han llamado mucho la atención de los investigadores; hay estudios que se centran en la importancia de diseñar rúbricas adecuadas con criterios de rendimiento claros, consistentes e integrales para garantizar un cierto grado de confiabilidad (Tierney y Simon, 2004; Brodie y Gibbins, 2009), o en la importancia de que los estudiantes tengan claridad objetivos a alcanzar (Szarka y Brestenska, 2012). También hay estudios que abordan la evaluación formativa (Couto, Durand, Wolff, Restini, Faria, Romão, y Bestetti, 2019), la autoevaluación (Lanthony, Azzouzi, François y Peyret, 2018) o la evaluación de los estudiantes por parte de los docentes (Claessens, 2019). El uso de rúbricas es útil no solo para los evaluadores, que tienen un estándar explícito, sino también para los estudiantes, porque proporcionan expectativas sobre lo que se evaluará (Szarka y 
Brestenska, 2012). Si los estudiantes saben claramente la forma en que serán evaluados, no solo pueden mejorar su desempeño, sino que también ganan confianza, ya que saben claramente lo que deben hacer para obtener buenas calificaciones. Además, las rúbricas también contribuyen a la evaluación formativa, ya que proporcionan comentarios y una marca (Brodie y Gibbins, 2019). Como apuntan Tierney y Simon (2004) las rúbricas de puntuación son especialmente útiles en la evaluación para el aprendizaje porque contienen descripciones cualitativas de los niveles de logro que funcionan bien dentro del proceso de evaluación (inicial, formativa y final), y especialmente la en la evaluación formativa. Destacaremos también que, si los diferentes criterios de evaluación se describen adecuadamente, una rúbrica de puntuación puede disminuir notablemente el grado de subjetividad en el proceso de evaluación, convirtiéndola en una herramienta útil para el profesorado. Si el alumnado tiene acceso a las rúbricas, que pueden presentarse de manera clara, por ejemplo, en forma de cuadrícula o tabla donde estén contenidos los diferentes estándares, pueden comparar en cualquier punto del proyecto el trabajo que están haciendo con los estándares que deben alcanzar, siendo de gran ayuda para el alumnado en su proceso de aprendizaje.

\section{Objetivos de la investigación}

Con el objetivo de investigar sobre la práctica de la evaluación en el ABP, el objetivo principal de esta investigación es conocer la opinión de los docentes de educación secundaria de lengua extranjera de la Región de Murcia sobre la evaluación dentro del Aprendizaje Basado en Proyectos (ABP). Para dar respuesta al siguiente objetivo general se han establecido los siguientes objetivos específicos con sus correspondientes hipótesis:

a. Conocer los recursos y técnicas de evaluación que se utilizan cuando trabajan por Aprendizaje Basados en Proyectos.

b. Determinar las diferencias existentes entre el profesorado según los datos sociodemográficos sobre el sistema de evaluación. Para lo cual consideramos la siguiente hipótesis: Existen diferencias estadísticamente significativas sobre el sistema de evaluación en $A B P$, en función de los datos sociodemográficos del profesorado participante.

\section{Investigación empírica}

\section{Metodología}

El diseño de este estudio es cuantitativo no experimental, con enfoque de corte descriptivo tipo encuesta porque, como dicen Cubo, Martín y Ramos (2011), el objetivo principal es describir de manera objetiva la situación de una parte de la comunidad educativa, en este caso, profesorado de educación secundaria en de la Región de Murcia. Para lograr este objetivo, hemos utilizado un cuestionario diseñado ad hoc, y optado por un diseño de tipo transversal, ya que la recogida de datos que se realiza en el grupo del profesorado en un solo momento temporal.

La población a la que va dirigida la investigación realizada está compuesta por profesorado de educación secundaria de la Región de Murcia. Para la selección de la muestra se ha utilizado la estrategia de muestreo no probabilístico casual o accidental cuya finalidad 
exploratoria trata de conocer las opiniones del profesorado acerca de la evaluación bajo el enfoque metodológico ABP. Las personas que participaron fueron un total de 43, a las que se les explicó la finalidad de la investigación y participaron voluntariamente, siguiendo los principios éticos regidos para este tipo de investigaciones.

Los datos sociodemográficos que aparecen descritos en la Tabla 1.

Tabla 1. Datos descriptivos y demográficos de la muestra

\begin{tabular}{|c|c|c|}
\hline Variable & Categorías & Estadísticos \\
\hline Sexo & Hombre & $34.9 \%(15)$ \\
\hline \multirow{5}{*}{ Edad } & Mujer & $65.1 \%(28)$ \\
\hline & 25 años o menos & $2.3 \%(1)$ \\
\hline & De 26 a 35 años & $27.9 \%(12)$ \\
\hline & De 36 a 45 años & $25.6 \%(11)$ \\
\hline & De 46 a 55 años & $34.6 \%(15)$ \\
\hline \multirow{5}{*}{ Experiencia laboral (años) } & Más de 55 años & $4 \%(4)$ \\
\hline & Menos de 3 años & $18.6 \%(8)$ \\
\hline & De 3 a 9 años & $25.6 \%(11)$ \\
\hline & De 10 a 19 años & $16.6 \%(7)$ \\
\hline & De 20 a 30 años & $25.6 \%(11)$ \\
\hline \multirow{4}{*}{ Titulación } & Más de 30 años & $14 \%(6)$ \\
\hline & Graduado en Filología Inglesa & $83.7 \%(36)$ \\
\hline & Graduado en Filología Francesa & $4.7 \%(2)$ \\
\hline & Graduado en otras especialidades & $33.8 \%(3)$ \\
\hline \multirow[b]{2}{*}{ Nivel en el que imparte docencia } & Graduado en Matemáticas & $4.7 \%(2)$ \\
\hline & ESO & $86 \%(37)$ \\
\hline \multirow[b]{2}{*}{ Tipo de centro } & Bachillerato & $14 \% 6)$ \\
\hline & Público & $74.4 \%(32)$ \\
\hline \multirow[t]{2}{*}{ Conocimientos de TIC } & $\begin{array}{l}\text { Concertado } \\
\text { Intermedio }\end{array}$ & $\begin{array}{l}25.6 \%(11) \\
79.1 \%(34)\end{array}$ \\
\hline & Avanzado & $30.9 \%(9)$ \\
\hline Formación en ABP & Inicial & $25.6 \%(11)$ \\
\hline
\end{tabular}




\begin{tabular}{lll}
\hline Variable & Categorías & Estadísticos \\
\hline & Intermedio & $41.9 \%(18)$ \\
& & \\
Situación laboral & Avanzado & $32.6 \%(14)$ \\
& Funcionario de carrera & $81.4 \%(35)$ \\
& & $18.6 \%(8)$ \\
\hline
\end{tabular}

En cuanto a las variables de esta investigación, la variable independiente corresponde al curso, edad, sexo y mención de los participantes, no obstante, se pondrá la atención en esta última, con el fin de dar respuesta a las hipótesis planteadas. En cuanto a la variable dependiente lo determina el grado de acuerdo o desacuerdo con los ítems, de modo que así se pueda establecer el nivel de conocimiento de los encuestados.

Para la recogida de información se elaboró expresamente el cuestionario conformado por dos apartados. La primera parte corresponde a los datos sociodemográficos. Y la segunda parta a los 20 ítems referidos a los procesos y sistemas de evaluación en ABP, presentando una escala de respuesta con cinco categorías donde 1 es «Totalmente en desacuerdo» y 5 es «Totalmente de acuerdo», tal cual queda reflejado en la Tabla 2.

Tabla 2-Dimensiones del cuestionario

\begin{tabular}{|l|l|c|}
\hline Dimensión & Descripción & Ítems \\
\hline Datos & Ítems para recoger algunos datos personales y académicos & 9 ítems \\
sociodemográficos & $\begin{array}{l}\text { sobre los cuestionados, tales como: sexo, edad, experiencia } \\
\text { laboral, titulación, nivel en el que imparte docencia, tipo de } \\
\text { centro, conocimientos TIC, formación en ABP y situación } \\
\text { laboral. }\end{array}$ & \\
\hline Software & $\begin{array}{l}\text { Ítems referidos a los procesos y sistemas de evaluación del } \\
\text { Aprendizaje Basado en Proyectos }\end{array}$ & 20 \\
\hline
\end{tabular}

Para la validación del contenido del cuestionario, se seleccionó a un grupo de cinco expertos compuesto por docentes especialistas en ABP, con el fin de conocer el grado en que el cuestionario presenta una muestra adecuada de los contenidos a los que se refiere, sin omisiones y sin desequilibrios de contenido siguiendo a Escobar Pérez y Cuervo Martínez (2008). Seguidamente, se realizó el test W de Kendall para analizar el grado de concordancia entre las puntuaciones emitidas por los expertos y el promedio de las puntuaciones asociadas a cada ítem para medir el grado de importancia y la adecuación de los mismos al estudio propuesto, determinando un alto grado de acuerdo. El nivel de fiabilidad del cuestionario fue analizado mediante el alfa de Cronbach (ODDwyer y Bernauer, 2014), obteniendo una puntuación global de 0.828. Este resultado indica una alta consistencia interna (De Vellis, 2003).

El análisis de los mismos se realizó con el paquete estadístico SPSS (Statistical Package forthe Social Sciences), versión 24. La naturaleza de las variables y los objetivos descritos permitieron aplicar las siguientes técnicas de análisis estadístico: Alfa de Cronbach para comprobar la fiabilidad del instrumento, cálculo de estadísticos descriptivos, así como la aplicación de pruebas de contraste de carácter no paramétrico, de acuerdo a los objetivos de 
la investigación, en concreto, $\mathrm{H}$ de Kruskal Wallis (contraste de k muestras independientes) y U de Mann Whitney (contraste de dos muestras independientes). Para la estadística inferencial se ha establecido un nivel de significación de $(\mathrm{p}<.05)$.

\section{Resultados}

En este apartado se presentan los resultados obtenidos organizados en función de los objetivos planteados.

Resultados relacionados con el Objetivo 1: Conocer los recursos y técnicas de evaluación que se utilizan cuando trabajan por Aprendizaje Basados en Proyectos

Los datos reflejados en la Tabla 3 muestran que el profesorado participante afirma que no asignan la misma calificación a todo el alumnado cuando el proyecto se realiza en colaboración $(\mathrm{M}=2.49)$ y que no se centran principalmente en los contenidos curriculares cuando evalúo $\mathrm{ABP}(\mathrm{M}=2.86)$, pero tampoco afirman con rotundidad que se centran en el resultado final del proyecto cuando evalúan un trabajo realizado con $A B P(M=3.53)$. En ese sentido, los docentes indican que explican a su alumnado la forma en que serán evaluados $(\mathrm{M}=4.53)$.

Tabla 3. Uso de las técnicas de evaluación de ABP por parte del profesorado

\begin{tabular}{|c|c|c|c|c|c|c|}
\hline \multirow[t]{2}{*}{ Ítem } & \multirow[t]{2}{*}{ Media } & \multicolumn{5}{|c|}{$\%$} \\
\hline & & 1 & 2 & 3 & 4 & 5 \\
\hline $\begin{array}{l}\text { 1. Me centro en el resultado final del proyecto cuando evalúo un } \\
\text { trabajo realizado con } A B P \text {. }\end{array}$ & 3.53 & 0.0 & 9.3 & 39.5 & 39.5 & 11.6 \\
\hline $\begin{array}{l}\text { 2. Me centro principalmente en los contenidos curriculares cuando } \\
\text { evalúo ABP. }\end{array}$ & 2.86 & 2.3 & 27.9 & 51.2 & 18.6 & 0.0 \\
\hline $\begin{array}{l}\text { 3. Utilizo la evaluación formativa en cada etapa del proceso para guiar } \\
\text { al alumnado a través del proyecto. }\end{array}$ & 3.86 & 0.0 & 4.7 & 23.3 & 53.5 & 18.6 \\
\hline $\begin{array}{l}\text { 4. Evalúo productos intermedios (planos, borradores, bocetos) y no } \\
\text { solo el resultado final. }\end{array}$ & 4.23 & 0.0 & 2.3 & 14.0 & 41.9 & 41.9 \\
\hline 5. Uso diferentes instrumentos para evaluar ABP. & 4.09 & 2.3 & 2.3 & 11.6 & 51.2 & 32.6 \\
\hline 6. Uso diferentes técnicas para evaluar ABP. & 3.88 & 2.3 & 2.3 & 25.6 & 44.2 & 25.6 \\
\hline $\begin{array}{l}\text { 7. Cada proyecto diferente requiere una forma de evaluación } \\
\text { individualizada. }\end{array}$ & 3.91 & 0.0 & 4.7 & 23.3 & 48.8 & 23.3 \\
\hline $\begin{array}{l}\text { 8. Utilizo un examen escrito para evaluar los aprendizajes del } \\
\text { alumnado después del proyecto. }\end{array}$ & 3.42 & 2.3 & 9.3 & 53.5 & 14.0 & 20.9 \\
\hline $\begin{array}{l}\text { 9. Evalúo } A B P \text { individualmente, incluso si el proyecto se realiza en } \\
\text { colaboración. }\end{array}$ & 3.95 & 0.0 & 4.7 & 16.3 & 58.1 & 20.9 \\
\hline $\begin{array}{l}\text { 10. Incluyo la evaluación del trabajo colaborativo en la evaluación de } \\
\text { ABP. }\end{array}$ & 4.00 & 0.0 & 4.7 & 16.3 & 53.5 & 25.6 \\
\hline $\begin{array}{l}\text { 11. Si el proyecto se realiza en colaboración, todo el alumnado obtiene } \\
\text { la misma calificación. }\end{array}$ & 2.49 & 16.3 & 37.2 & 30.2 & 14.0 & 2.3 \\
\hline 12. Evalúo la creatividad al evaluar ABP. & 4.09 & 0.0 & 2.3 & 16.3 & 51.2 & 30.2 \\
\hline $\begin{array}{l}\text { 13. Cambio el plan de evaluación durante ABP para acomodar cambios } \\
\text { en el proyecto. }\end{array}$ & 3.84 & 0.0 & 2.3 & 32.6 & 44.2 & 20.9 \\
\hline
\end{tabular}




\begin{tabular}{|c|c|c|c|c|c|c|}
\hline \multirow[t]{2}{*}{ Ítem } & \multirow[t]{2}{*}{ Media } & \multicolumn{5}{|c|}{$\%$} \\
\hline & & 1 & 2 & 3 & 4 & 5 \\
\hline 14. Creo rúbricas a medida para evaluar $\mathrm{ABP}$. & 4.16 & 0.0 & 0.0 & 16.3 & 51.2 & 32.6 \\
\hline 15. Explico al alumnado la forma en que serán evaluados. & 4.53 & 0.0 & 0.0 & 7.0 & 32.6 & 60.5 \\
\hline 16. Incluyo una parte de autoevaluación al final de los proyectos. & 3.88 & 2.3 & 14.0 & 16.3 & 27.9 & 39.5 \\
\hline 17. Incluyo una parte de autoevaluación durante los proyectos. & 3.42 & 7.0 & 11.6 & 34.9 & 25.6 & 20.9 \\
\hline 18. Incluyo evaluación por pares sobre el producto final. & 3.53 & 7.0 & 2.3 & 37.2 & 37.2 & 16.3 \\
\hline $\begin{array}{l}\text { 19. Incluyo una evaluación inicial para compararla con la evaluación } \\
\text { final. }\end{array}$ & 3.30 & 7.0 & 11.6 & 44.2 & 18.6 & 18.6 \\
\hline 20. Incluyo la evaluación de habilidades orales en la evaluación de ABP. & 4.28 & 0.0 & 0.0 & 14.0 & 44.2 & 41.9 \\
\hline
\end{tabular}

Resulta interesante comprobar que, según reconocen las docentes, usan diferentes formas de evaluar cuando trabajan con ABP (M=4.09), toda vez que reconocen, incluyen además la evaluación del trabajo colaborativo que conlleva la metodología de ABP $(M=4.0)$. Finalmente, atendiendo a los datos, el profesorado participante señala que evalúa los productos intermedios (planos, borradores, bocetos) y no solo el resultado final $(\mathrm{M}=4.23)$ y que trabajando con ABP evalúa las habilidades orales del alumnado $(\mathrm{M}=4.28)$.

Los resultados encontrados muestran que hay aspectos que sobre los que el profesorado no tiene una opinión clara. No emiten una respuesta contundente en cuanto al uso una prueba escrita para evaluar los aprendizajes de los estudiantes después del proyecto $(\mathrm{M}=3.42)$, lo mismo ocurre cuando se les pregunta si realizan una evaluación inicial para compararlo con la evaluación final $(\mathrm{M}=3.30)$.

Resultados relacionados con el Objetivo 2: Determinar las diferencias existentes entre el profesorado según los datos sociodemográficos sobre el sistema de evaluación

Para dar respuesta a este objetivo, hemos procedido a comprobar la hipótesis H1: Existen diferencias estadísticamente significativas sobre el sistema de evaluación en $A B P$, en función de los datos sociodemográficos del profesorado participante, se ha utilizado estadística no paramétrica.

Tras el cálculo de la prueba estadística U de Mann-Whitney con la variable sexo, se encuentran diferencias estadísticamente significativas en el ítem Incluyo la evaluación del trabajo colaborativo en la evaluación de $A B P$ y en el ítem Creo rúbricas a medida para evaluar el $A B P$ favorables a las docentes. Tras aplicar la prueba Kruskal Wallis con la edad como la variable de agrupación, se aprecian diferencias estadísticamente significativas en el ítem Creo rúbricas personalizadas para evaluar $A B P$, favorables al profesorado más joven. Cuando tomamos como variable de contraste la experiencia docente, los resultados de la prueba de Kruskal Wallis arrojan diferencias estadísticamente en el ítem cuando el proyecto se realiza en colaboración, todos los estudiantes obtienen las mismas calificaciones, favorables al profesorado con menos experiencia. Con el resto de variables sociodemográficas no se han apreciado diferencias significativas. 


\section{Discusión}

Los resultados obtenidos han dejado clara evidencia que para el profesorado supone un reto evaluar cuando se trabaja con ABP, en la línea de Giabbanelli y Tawfik (2017). Si bien, los docentes participantes reconocen que cuando trabajan con ABP evalúan algo más que los contenidos curriculares y, por tanto, plantean técnicas e instrumentos de evaluación ajustados a la idiosincrasia de esta metodología, coincidiendo así con Gentry (2000), Sa et al. (2019) y Shafirova y Cassany (2017) en que el reto de la evaluación cuando se utiliza ABP es establecer técnicas de valoración que contemplen aspectos relacionados con el desarrollo de los proyectos y la participación del alumnado. Aunque si bien, y en contraposición de Gentry (2000) y Thomas (2000) que abogan la utilización de variadas técnicas de evaluación y en diferentes momentos del proceso de enseñanza y aprendizaje, hay aspectos que no consideran, como es el caso de la comparativa entre lo que los alumnos saben antes de iniciar el proceso de aprendizaje y al finalizar el mismo, realizando evaluaciones iniciales y finales para recoger los datos y comprobar de manera más eficaz el efecto del proceso de enseñanza y de la asimilación y comprensión de los contenidos trabajados en el aula.

En cuanto a las calificaciones, los docentes participantes señalan que el hecho de trabajar actividades realizadas de forma colaborativa no conlleva la misma calificación para todo el componente del grupo, lo cual se ve favorecido por el uso de rúbricas y otros instrumentos de evaluación, así como la explicación de las calificaciones al alumnado; en esto podemos observar que hay coincidencia con Gentry (2000) y Thomas (2000), en cuanto a la utilización de formas de evaluación variada, así como el uso de instrumentos determinados de evaluación como las rúbricas tal y como plantean Brodie y Gibbins, (2009) y Tierney y Simon (2004).

Además, esta investigación pone de relieve que el profesorado que trabaja por proyectos le otorga mucha importancia a que el alumnado sepa exactamente la forma en que será evaluado. Szarka y Brestenska (2012) enfatizan la importancia de tener pautas claras de evaluación para el alumnado, cuando dicen que esas pautas tienen un efecto motivador al tiempo que aumentan la autoconfianza y la autoconciencia en los estudiantes. Todo ello contribuye a la evaluación formativa del alumnado, proporcionándoles la conveniente retroalimentación (Brodie y Gibbins, 2019; Tierney y Simon, 2004).

Los resultados indican que el profesorado de la muestra considera relevante diferentes aspectos de la evaluación en ABP como es la evaluación del proceso, y del uso de diferentes instrumentos para evaluar estos elementos. En este sentido, se hace eco Gentry (2000) cuando señala que la evaluación en ABP ha de plantificar cómo evaluar el cumplimiento de los objetivos a corto y largo plazo y ha de tener presente la capacidad del alumnado para trabajar como parte de un equipo.

La creación de rúbricas a medida del proyecto también es un elemento transcendental para la mayoría del profesorado; a la vez que es un elemento que presenta diferencias relacionadas con los datos sociodemográficos, ya que las mujeres y el profesorado más joven otorga más importancia a las rúbricas como instrumentos de evaluación en ABP, coincidiendo con los estudios de Brodie y Gibbins (2019). 


\section{Conclusión}

Concluimos diciendo que la evaluación de las tareas de ABP, entre las que se encuentran las evaluaciones relacionadas con la búsqueda de información e investigación, la capacidad de síntesis, la colaboración y el trabajo en grupo, la gestión y la planificación de los contenidos y su transferencia, así como la creación de un producto final con toda la información recogida y organizada; es, por lo tanto, un proceso complejo, que debiera conllevar la evaluación de cada una de las tareas realizada, pues de esta manera se garantiza de manera rigurosa la evaluación de todo un proceso de enseñanza y aprendizaje donde se pueden valorar las complejas estrategias cognitivas desarrolladas por el alumnado; y que difieren de las estrategias que se desarrollan en aquellos sistemas de enseñanza socráticos donde el alumno es expuesto a los contenidos de forma pasiva y su contribución consiste en practicarlos en tareas que implican la repetición o el recuerdo de lo explicado, y no la creación de algo nuevo a partir de dichos contenidos, como sucede en el ABP. Por otro lado, es importante dejar constancia que muchos de los elementos que el profesorado debe evaluar en ABP no son fácilmente evaluables, y en muchos casos son altamente subjetivos; por ello, para lograr una buena evaluación en el ABP, se deben incluir diferentes métodos e instrumentos de evaluación, teniendo en cuenta las diferentes partes del proceso de enseñanza y aprendizaje y las múltiples habilidades que se desean medir. Con el objetivo de reducir el nivel de subjetividad mencionado, se deben usar rúbricas específicas en cada producto que se debe evaluar, para tener una guía clara de los estándares y criterios que se desean valorar. Aunque podemos concluir que no es una tarea sencilla, lograr una buena evaluación de los proyectos de ABP, sin embargo, no debe ser tampoco una tarea difícil e insalvable, y para facilitar este proceso, el profesorado debe ampliar su espectro de evaluación, poniendo su punto de mira en los aspectos relevantes relacionados tanto con la evaluación del proceso de aprendizaje y como de los contenidos adquiridos para lograrlo. Una buena evaluación del ABP es una gran estrategia educativa tanto para que el profesorado, como para el alumnado, pues de esta manera obtendrán información sobre los logros conseguidos con el abordaje de los diferentes proyectos diseñados.

Para finalizar decir que este estudio sienta las bases de futuras investigaciones sobre sobre qué instrumentos son más eficaces para evaluar los logros del alumnado durante todo el proceso de enseñanza y aprendizaje en el ABP.

\section{Referencias}

Brodie, L., \&Gibbings, P. (2009). Comparison of PBL assessment rubrics. Research in Engineering Education Symposium (REES). Cairns, QLD, Australia.

Carrillo-García, M.E., \&Cascales-Martínez, A. (2016). Cuestionario sobre el Aprendizaje Basado en Proyectos en Educación Infantil y Primaria según el profesorado (CABPEIB). Recuperado de https://cutt.ly/6e2iLpm 
Cascales-Martínez, A., \& Carrillo-García, M. E. (2018). Aprendizaje basado en proyectos en educación infantil: cambio pedagógico y social. Revista Iberoamericana De Educación, 76, 79-98. Doi: https://doi.org/10.35362/rie7602861

Cascales-Martínez, A., Carrillo-García, M., \& Redondo-Rocamora, A. M. (2017). ABP y tecnología en Educación Infantil. Pixel-Bit. Revista de Medios y Educación, 50, 201210.

Claessens, S. (2019). The role of student evaluations in a PBL centred law curriculum: towards a more holistic assessment of teaching quality. The Law Teacher. 1-12. Doi: https://doi.org/10.1080/03069400.2019.1580009

Couto, L., Durand, M., Wolff, A., Restini, C., Faria Jr., M., Romão, G., \&Bestetti, R. (2019) Formative assessment scores in tutorial sessions correlates with OSCE and progress testing scores in a PBL medical curriculum. Medical Education Online, 24:1, 1560862.Doi: https://doi.org/10.1080/10872981.2018.1560862

Cubo, S., Martín, B. \& Ramos, J.L. (2011). Métodos de investigación y análisis de datos en ciencias sociales y de la salud. Madrid: Pirámide.

De Vellis, R.F. (2003) (2a. ed.). Scale development: Theory and applications. ThousandOaks CA: Sage.

Escobar Pérez, J., \& Cuervo Martínez, A. (2008). Validación de contenido y juicio de expertos: una aproximación a su utilización. Avancesenmedición, 6(1), 27-36.

Gentry, E. (2000). Creating Student-centered, Problem-based Classrooms. Huntsville: University of Alabama.

Giabbanelli, P., \& Tawfik, A. (2017). Overcoming the PBL assessment challenge: Design and development of the incremental thesaurus for assessing causal maps (ITACM). Technology, Knowledge and Learning. Doi: https://doi.org/10.1007/s10758-0179338-8

Lanthony, A., Azzouzi, E., François, A., Peyret, N. (2018). Self-assessment in PBL: A tool to develop self-confidence and autonomy of students: The example of self-assessment experiment at ISMEP-Supmeca in France IEEE Global Engineering Education Conference (EDUCON). Doi: https://doi.org/10.1109/EDUCON.2018.8363238

O’Dwyer, L. y Bernauer, J. (2014). Quantitative Research for the Qualitative Researcher. Thousand Oaks. California: Sage

Sa, B., Ezenwaka, C., Singh, K., Vuma, S., \& Majumder, A. (2019). Tutor assessment of PBL process: does tutor variability affect objectivity and reliability? BMC Medical Education, 19:76. Doi: https://doi.org/10.1186/s12909-019-1508-z 
Shafirova, L., \& Cassany, D. (2017). Aprendiendo idiomas en línea en el tiempo libre. Revista de estudios socioeducativos. ReSed, (5), pp. 49-62. Recuperado de https://cutt.ly/Ue2iKKJ

Szarka, K., \& Brestenska, B. (2012). Implementation the assessment rubrics to evaluate the outcomes of PBL and ABL process. ICETA 2012 - 10th IEEE International Conference on Emerging eLearning Technologies and Applications, Proceedings. 377-380. Doi: https://doi.org/10.1109/ICETA.2012.6418301.

Tobón, S. (2006). Competencias, calidad y educación superior. Coop. Editorial Magisterio.

Tierney, R., \& Simon, M. (2004). What's still wrong with rubrics: Focusing on the consistency of performance criteria across scale levels. Practical Assessment, Research \& Evaluation. 9(2). 1-7

Thomas, J. W. (2000). A Review of Research on Project-Based Learning. Recuperado de https://cutt.ly/8e2iJ88

Utech, J. (2003). Problem-Based Learning in the Student Centered Classroom. Recuperado de https://cutt.ly/Ee2iJk 